\title{
A Proposal to Integrate the POSIX Execution-Time Clocks into Ada 95^
}

\author{
J. Miranda ${ }^{1}$ and M. González Harbour ${ }^{2}$ \\ jmiranda@iuma.ulpgc.es, mgh@unican.es \\ 1 Applied Microelectronics Research Institute, Univ. Las Palmas de Gran Canaria \\ 35017 Las Palmas de Gran Canaria, SPAIN \\ 2 Departamento de Electrónica y Computadores, Univ. Cantabria \\ 39005 - Santander, SPAIN
}

\begin{abstract}
In this paper we present a proposal to integrate the POSIX.1 executiontime clocks and execution-time timers into the Ada 95 language. This proposal defines a new package named Ada.CPU_Time and describes the modifications done to the GNAT front-end and run-time to support it. Additionally this proposal discusses some usage schemes of this new interface.
\end{abstract}

Keywords: Scheduling, Hard Real-Time, Ada 95, Execution-Time, GNAT.

\section{Introduction}

Traditional real-time systems were built (and still are) using executive schedulers[4]. In these systems, if a particular task or routine exceeded its budgeted execution time, the system could detect the situation. Basically, whenever the minor cycle interrupt came in, it could check whether the current action had completed or not. If not, that meant an overrun. Unfortunately, in concurrent real-time systems built with multitasking preemptive schedulers, there is no equivalent method to detect and handle execution-time overruns. This is the case for systems built using the Ada tasking model and the associated Real-Time Annex[9].

In addition to detecting budget overruns, many flexible real-time scheduling algorithms require the capability to measure execution time and be able to perform scheduling actions when a certain amount of execution time has been consumed (for example, sporadic servers in fixed priority systems, or the constant bandwidth server in EDFscheduled systems). Support for execution-time clocks and timers is essential to be able to implement such flexible scheduling algorithms.

In recognition of all these application requirements, the Real-Time extensions to POSIX have recently incorporated support for them. Real-Time POSIX supports execution-time clocks and timers that allow an application to monitor the consumption of execution time by its tasks, and to set limits for this consumption. The next revision of

\footnotetext{
* This work has been partially funded by the Spanish Research Council (CICYT), contract numbers TIC2001-1586-C03-03 and TIC99-1043-C03-03, and by the Commission of the European Communities under project IST-2001-34820 (FIRST).
} 
the Ada language should support this functionality in order for the language to continue to be the best for programming real-time applications.

There has been a previous proposal to include execution-time clocks and timers into the Ada language $[6,3]$. That proposal defines a new package that includes all the operations required to access the execution-time monitoring functionality. Some of those operations belong to a protected object type that represents execution-time timers. That approach does not require modification of the compiler, nor of the run-time system (provided that the POSIX execution-time functionality is available), but its executiontime type is not integrated in the Ada language as a Time type.

In this paper we present a proposal to integrate the POSIX.1 execution-time clocks and timers into the Ada 95 language. In this proposal we try to minimize the implementation impact on the compiler and run-time system, and therefore we will not include any new language construct. It is composed of a new package named Ada.CPU_Time and the proposed modifications to the GNAT[5] front-end and run-time to efficiently support the execution-time clocks and timers. The proposal is being submitted to the Ada Rapporteur Group (ARG) for possible inclusion in a future revision of the Ada standard.

This paper is organized as follows. In Section 2 we present the interface of our proposed Ada.CPU_Time Ada package. In Section 3 we present five basic usage schemes of the execution-time clocks and timers. In Section 4 we briefly present the modifications done to the GNAT compiler to allow the use of execution-time timers in the Ada 95 timed sentences. We close with some conclusions and references.

\section{Ada.CPU_Time}

Our proposed interface to handle CPU_Time is based on the standard Ada.Real_Time interface. The major differences between our Ada.CPU_Time interface and the standard Ada.Real_Time interface are:

- The new data type Clock_Id is used to represent execution-time clocks. A value of this type represents the execution-time clock of a given task. According to the POSIX definition of execution time[7], it is implementation defined to whom will be charged the effects of interrupt handlers and run-time services on behalf of the system.

- The Time type represents absolute values of execution time as measured by a given execution-time clock. Values of this type have an internal Clock_Id value that ties the value of execution time to its associated clock. If a variable of type Time is not initialized, the value of its internal Clock_Id is undefined. The type Time is a time type as defined by ARM95, Section 9.6, and thus values of this type may be used in a delay_until_statement.

- The Time_Span type represents length of execution-time duration, and its values are not dependent upon any particular execution-time clock (or task).

- The new function CPU_Clock is used to get the execution-time clock identifier associated with each Ada task. 
- The function Clock has a new parameter used to specify the execution-time clock to be read. In order to keep compatibility with the Ada Calendar and Real_Time packages, if no parameter is passed then the execution-time clock of the calling task is returned.

- The new function Clock_Id_Of returns the identifier of the execution-time clock associated with the Time parameter $\mathrm{T}$.

- The function Time_Of has a new parameter used to associate the time to an executiontime clock.

- The exception Time_Error is raised by the function Clock if the Clock_Id parameter is not valid. This exception is also raised by operators "+" and "-", and the function Clock_Id_Of, if an execution-time parameter is not valid (for example, if it is not initialized). In addition, it is also raised by a delay_until_statement if a Time value corresponding to the task executing the statement is used, because otherwise this situation would cause a deadlock.

- The exception Incompatible_Times is raised by operator "-" if the execution-time parameters correspond to different execution-time clocks.

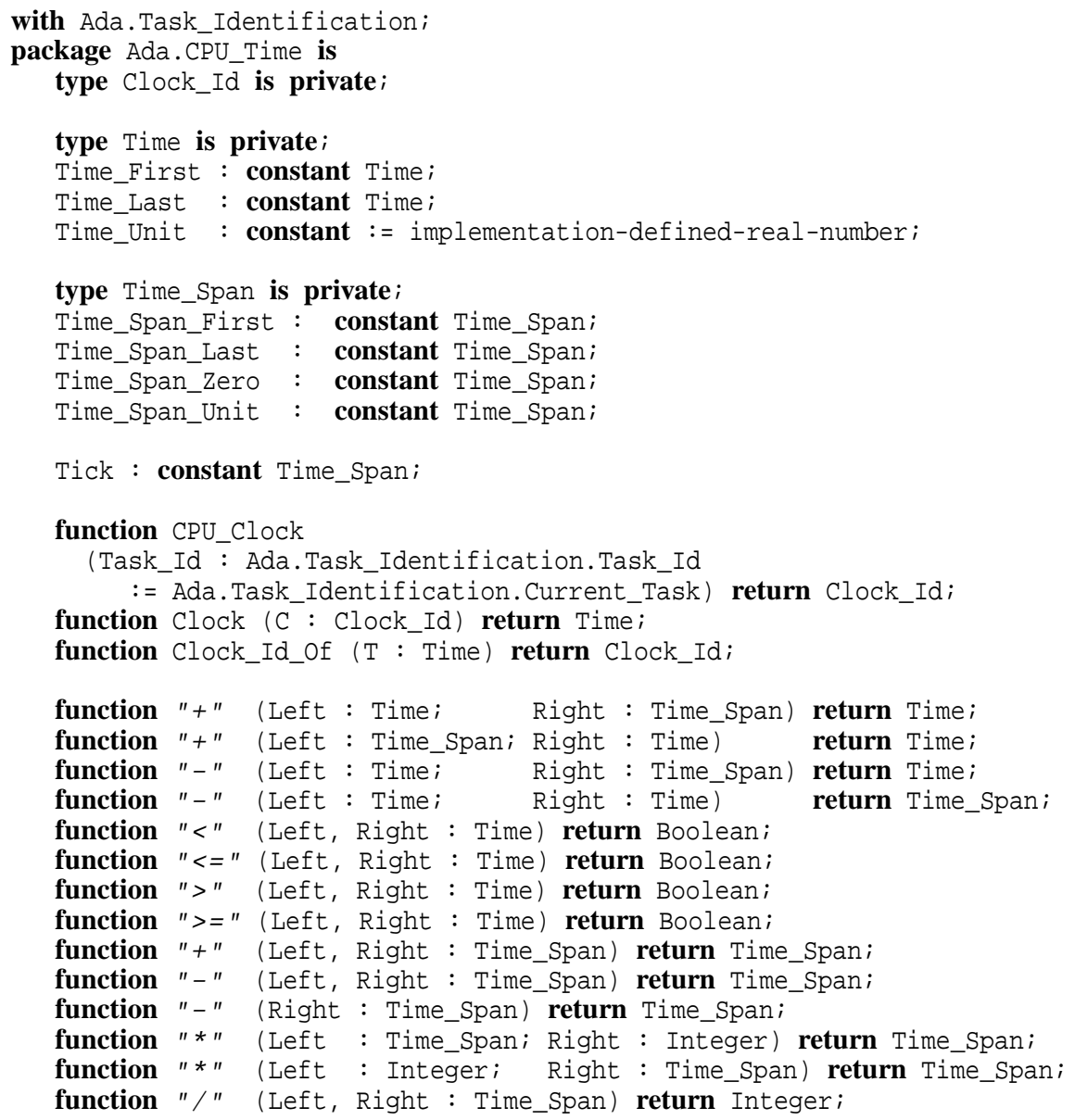




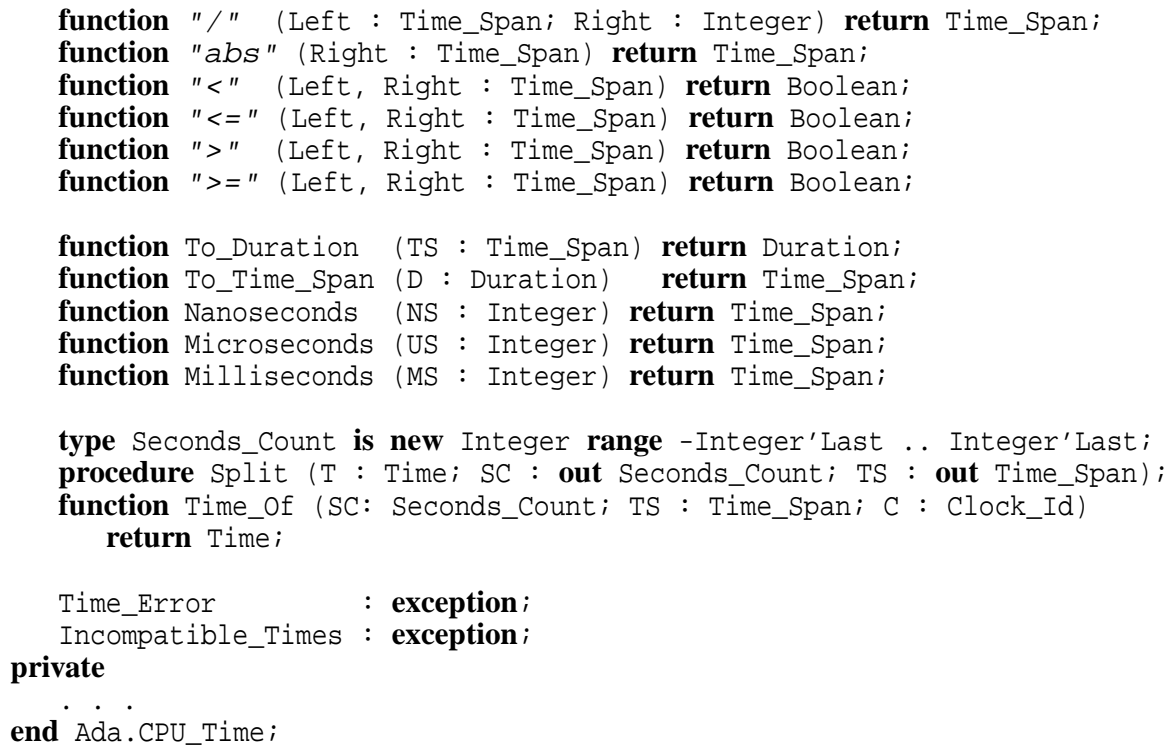

\section{Usage Schemes for Ada.CPU_Time Timers}

Using package Ada.CPU_Time, described above, we can design some basic usage schemes that depend on the particular needs of the application task whose execution time is being monitored. We have identified five major schemes:

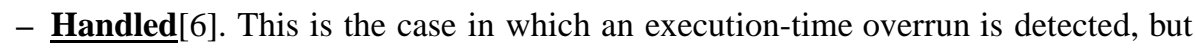
the task is allowed to complete its execution. This is applicable to systems under testing, or for tasks that have a high degree of critically (an thus cannot be stopped) or for which an occasional execution-time overrun can be tolerated, but needs to be reported.

In this scheme, the application task uses a single variable $\mathrm{T}$ to remember the value of the execution-time clock and to evaluate the execution time of the work. If the execution time is higher than MAX_TIME then it handles the execution-time error.

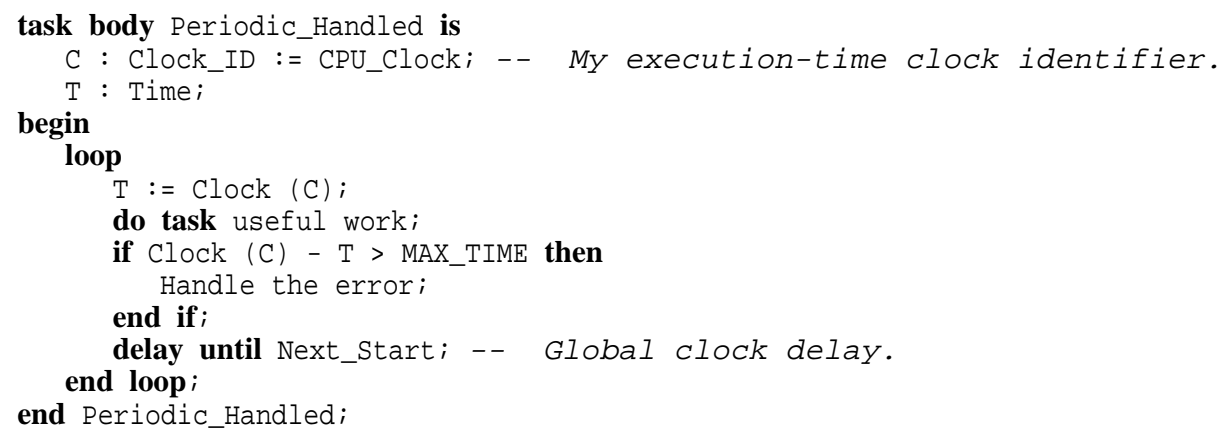


- Priority change[6]. In this scheme when the overrun is detected the priority of the task is lowered or increased (depending on the application requirements). A simple implementation of this scheme uses two nested tasks: the Worker task and the Supervisor task. The Supervisor task sleeps until the execution time of the Worker task reaches the instant of the priority change. When this happens the supervisor lowers (or increases) the priority of the worker task. If the Worker task completes the work before the instant of the priority change then it aborts the Supervisor task.

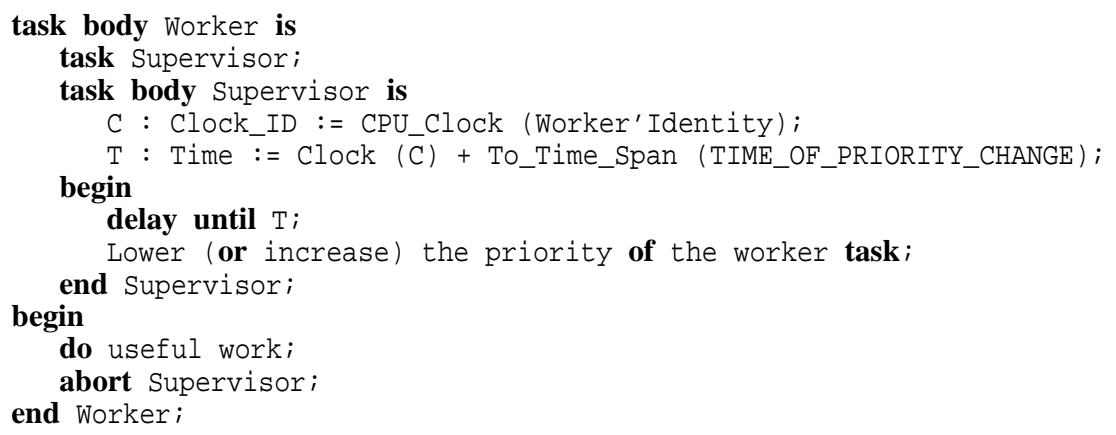

An alternative implementation of this scheme does not require abort. It can be done by means of the Ada select statement and one entry (i.e, Work_Done). When the worker completes its work calls Work_Done. The supervisor can then be implemented by means of a timed selective accept. If the entry call is received before the instant of priority change is reached then the work has been successfully completed in time; otherwise the time-budget has expired and the Supervisor lowers (or increases) the priority of the worker task. In addition the Supervisor task must accept the call to Work_Done that will be issued by the Worker at the end of its work.

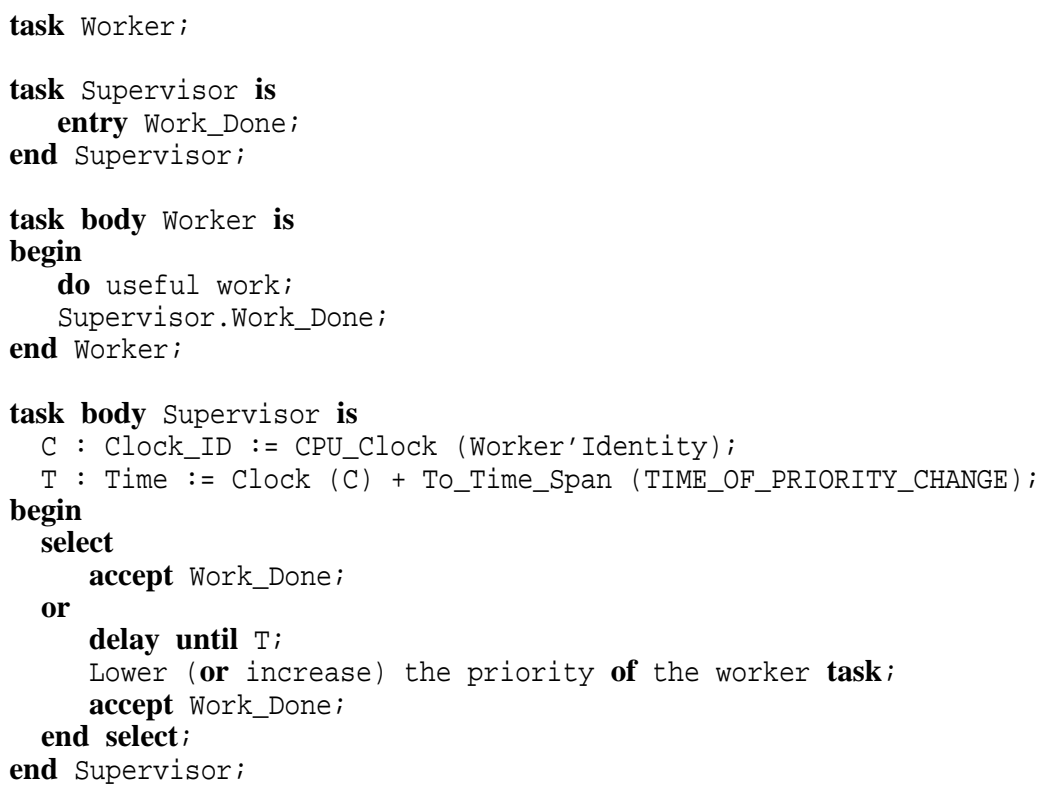


- Stopped[6]. This is the case in which if an execution-time overrun is detected, the associated task execution is stopped to allow lower priority tasks to execute within their deadlines. The task itself waits until its next activation and then proceeds normally.

In the implementation of this scheme an asynchronous select statement is used to abort the task's work if an execution-time overrun is detected.

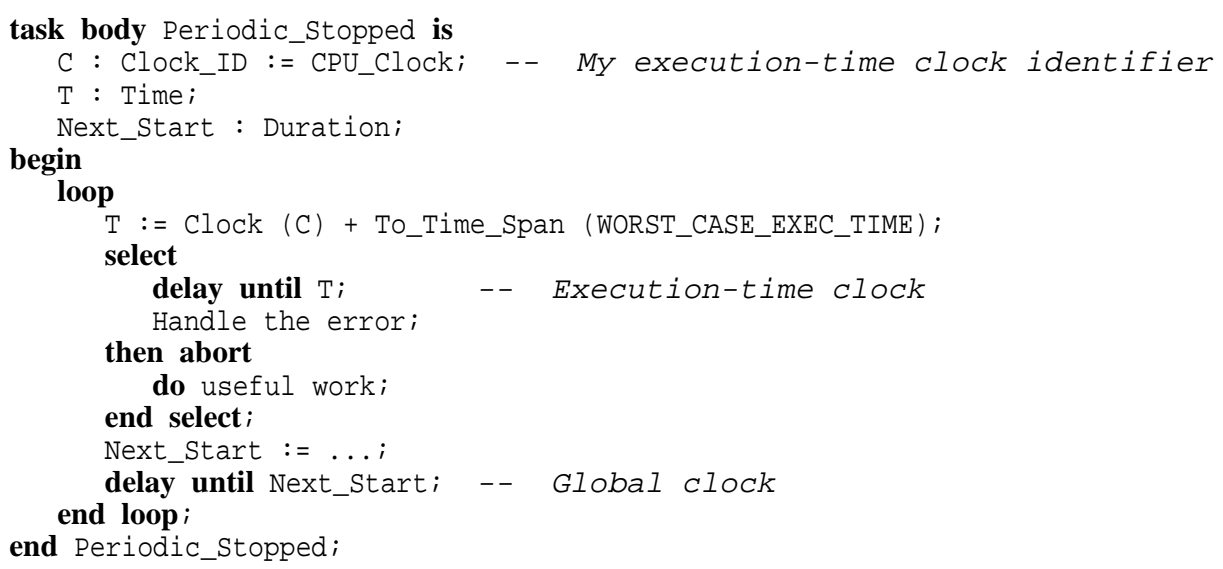

- Imprecise[6]. This scheme corresponds to the case in which the task is designed using the imprecise computation model[10], in which the task has a mandatory part (generally short and for which it is easier to estimate a worst-case execution time), and an optional part that refines the calculations made by the task. Since the worstcase execution time of this optional part is usually more difficult to estimate, this part will be aborted if an execution-time overrun is detected. This technique is also valid for cases in which the optional part continuously refines the quality of the results; we can let the optional part run until it exhausts its execution-time budget, and then use the last valid result obtained.

The implementation of this scheme consists of using the "handled" approach for the mandatory part of the task, and the "stopped" approach for the optional part. After the optional part, whether it is aborted or not, another mandatory part may exist to cause outputs of the task to be generated. Therefore this scheme can be implemented as follows:

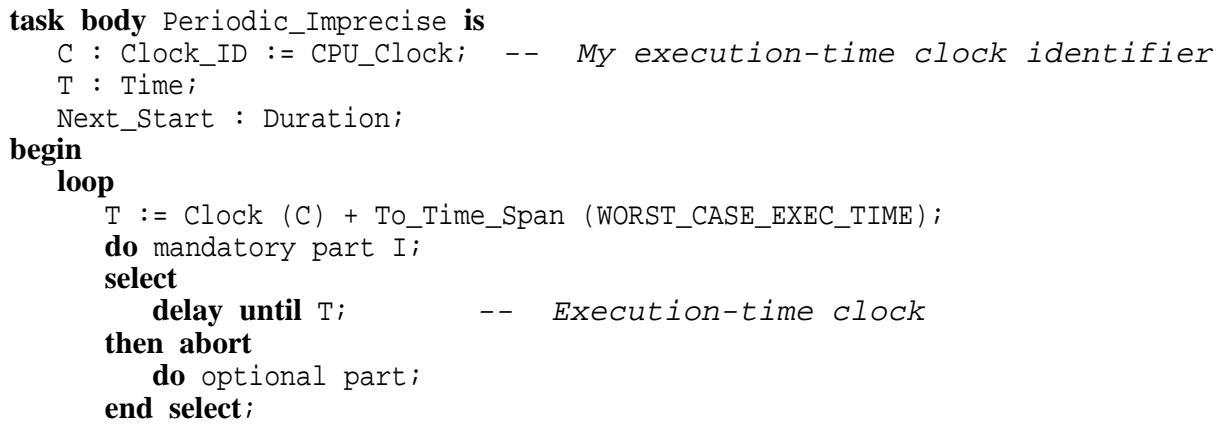




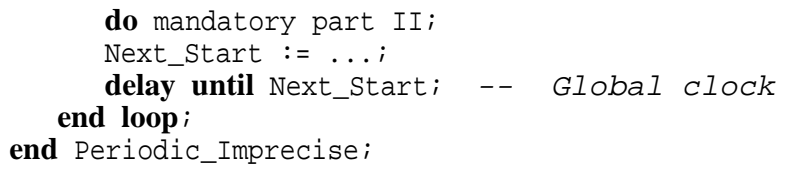

\section{Detailed Description of the Integration of the Execution-Time Timers into the GNAT Compiler}

In this section we describe the modifications done to the GNAT compiler to support the POSIX execution-time timers in Ada. For each Ada timing statement (delay until, timed entry call, and timed selective accept) we present the modifications done to the GNAT front-end and run-time.

\subsection{Delay Until Statement}

\section{Front-end}

- Semantics. The subprogram Analyze_Delay_Until has been modified to allow the use of the Ada.CPU_Time.Time type in the Ada 95 delay until statement.

- Expander. When an Ada.CPU_Time.Time type variable is used to specify the timeout, the expander has been modified to transform the delay until statement as follows:

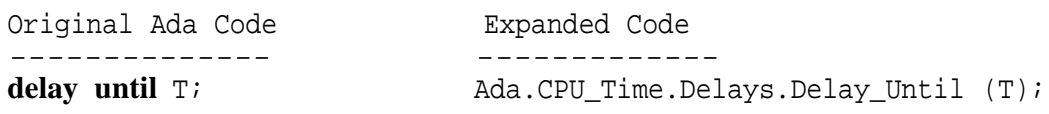

\section{Run-Time}

- Package System.Tasking. A POSIX execution-time timer and two flags have been added to the Ada Task Control Block (ATCB). The flags are used to remember if the timer has been created (and therefore the execution-time timer field is valid), and if the ATCB timer is currently in use.

- Package Ada.CPU_Time.Delays. The subprogram Delay_Until has been programmed to do the following actions:

1. Defer the abortion of the calling task.

2. Lock the ATCB of the calling task.

3. If the ATCB has not been created then create, program, and arm the ATCB execution-time timer; if the ATCB had been created but it is not in use then program and arm the ATCB execution-time timer; otherwise create, program and arm a new execution-time timer. In all these cases the address of a Delay Block register composed of the following fields is associated with the execution-time timer:

- The ATCB address of the calling task. 
- A boolean field (Timed_Out) initialized to false. This field will be used by the timed statements to differentiate the case of the timeout expiration from the case in which the blocked task is awakened by some other task (i.e. the acceptor of a timed entry call, or the caller of a selective wait).

4. Pass the calling task to the Delay_Sleep state.

5. Stop the calling task until the timeout expires. This is done by blocking the calling task using the caller mutex and condition variable declared by GNAT in the ATCB for this purpose.

6. Pass the calling task to the Runnable state.

7. If the ATCB timer was re-used then mark it as "not in use". Otherwise, remove the execution-time timer.

8. Unlock the ATCB of the calling task.

9. Verify if a request to abort the calling task has been received during this delay. If true then abort the task; otherwise undefer its abortion.

A task is used to handle the signal associated with all the execution-time timers. This task does following actions:

1. Block all the signals.

2. Activate the signal associated with all the execution-time timers.

3. Await for the execution-time timers signal.

4. Get the address of the Delay Block register associated with the execution-time timer.

5. Set to True the field Timed_Out of this Delay Block register.

6. Awaken the task that programmed this execution-time timer.

7. Go to step 3 .

Behavior The calling task programs an execution-time timer and becomes blocked until this timer expires.

\subsection{Timed Entry Call}

\section{Front-end}

- Semantics. No modification was required.

- Expander. When an Ada.CPU_Time.Time variable is used to specify the timeout the expander has been modified to transform the Ada timed entry call statement in the following way:

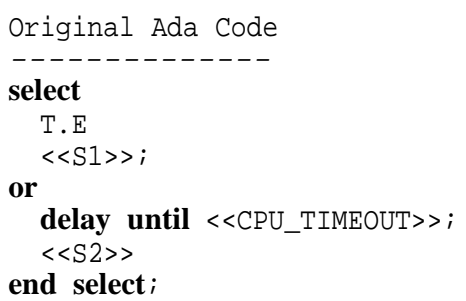

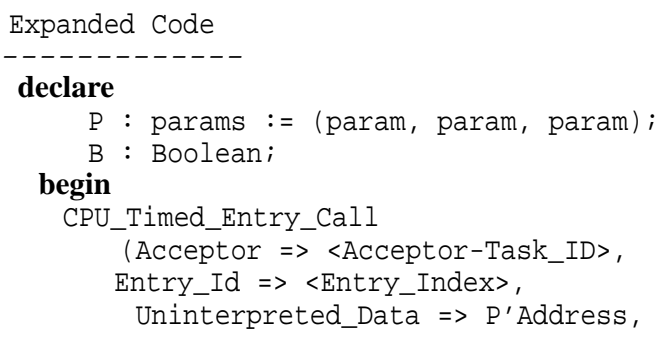




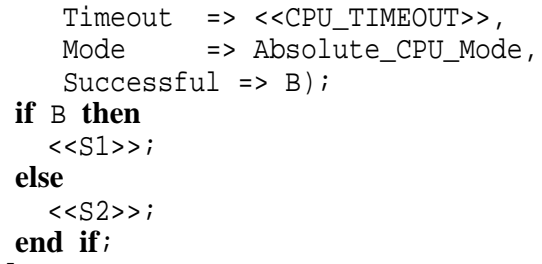

\section{Run-Time}

- Package System.Tasking.Rendezvous The new CPU_Timed_Entry_Call subprogram is based on the GNAT Timed_Entry_Call subprogram. The main differences with the original GNAT version are:

- The data type of the Timeout parameter is Ada.CPU_Time.Time (instead of Duration).

- Its body calls CPU_Wait_For_Completion_With_Timeout instead of the GNAT Wait_For_Completion_With_Timeout version.

- Package System.Tasking.Entry_Calls The new subprogram CPU_Wait_For_Completion_With_Timeout is based on the GNAT subprogram Wait_For_Completion_With_Timeout. The only difference is that it calls CPU_Timed_Sleep (instead of the GNAT subprogram Timed_Sleep).

- Package System.Task_Primitives.Operations The new subprogram Ada.CPU_Timed_Sleep calls Ada.CPU_Time.Delays.Delay_Until.

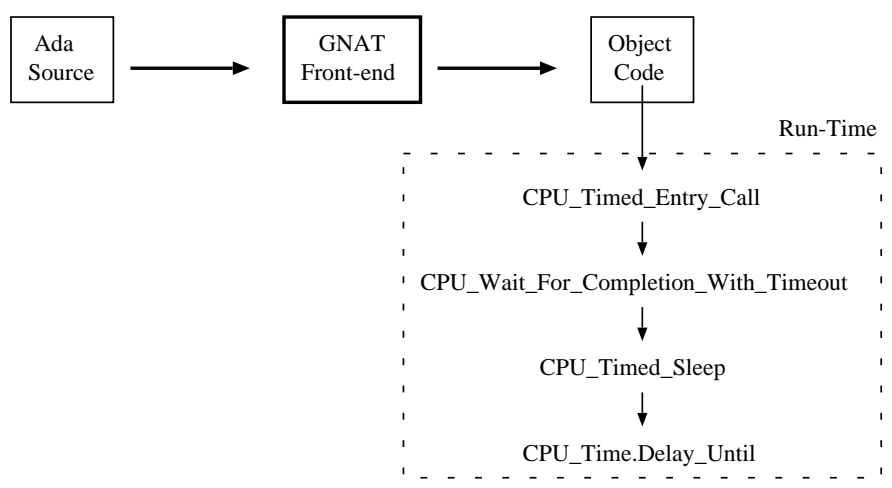

Fig. 1. Run-Time Calls to Implement the CPU Timed Entry Call.

Behavior. If the rendezvous can be immediately accepted the subprogram CPU_TimedEntry_Call completes the rendezvous and returns True in the out mode parameter Successful. Otherwise it programs an execution-time timer by calling the subprogram Ada.CPU_Time.Delays.Delay_Until. 
Behavior First of all let's briefly explain the semantics of the GNAT “at end" statement. It is a handler which provides a common way out of a block of statements even when an exception is propagated.

In the above code, after the execution-time timer is armed the abortable part is executed. If the abortable part completes its execution before the execution-time timer expires then the Async_Cancel_Timer is called to disarm the timer. Otherwise the executiontime timer expires and the signal catcher calls the run-time subprogram Locked_Abort_To_Level which defers the abortion of the blocked task, and cancels all the nested ATC (if any) done in the abortable part by raising the internal exception _abort_signal. After the abortion is undefered (in the exception handler) if the timeout had expired then the block of statements $<<S 1>>$ is executed.

\subsection{Timed Selective Accept}

\section{Front-end}

- Semantics. It has been modified to disallow the simultaneous use of Ada.CPU_Time and Ada.Real_Time time variables to specify multiple timeouts in the Ada 95 timed selective accept ${ }^{3}$.

- Expander. When the Ada.CPU_Time time variable is used to specify the timeouts of the following Ada 95 timed-selective statement the GNAT expander has been modified to generate the following block of code:
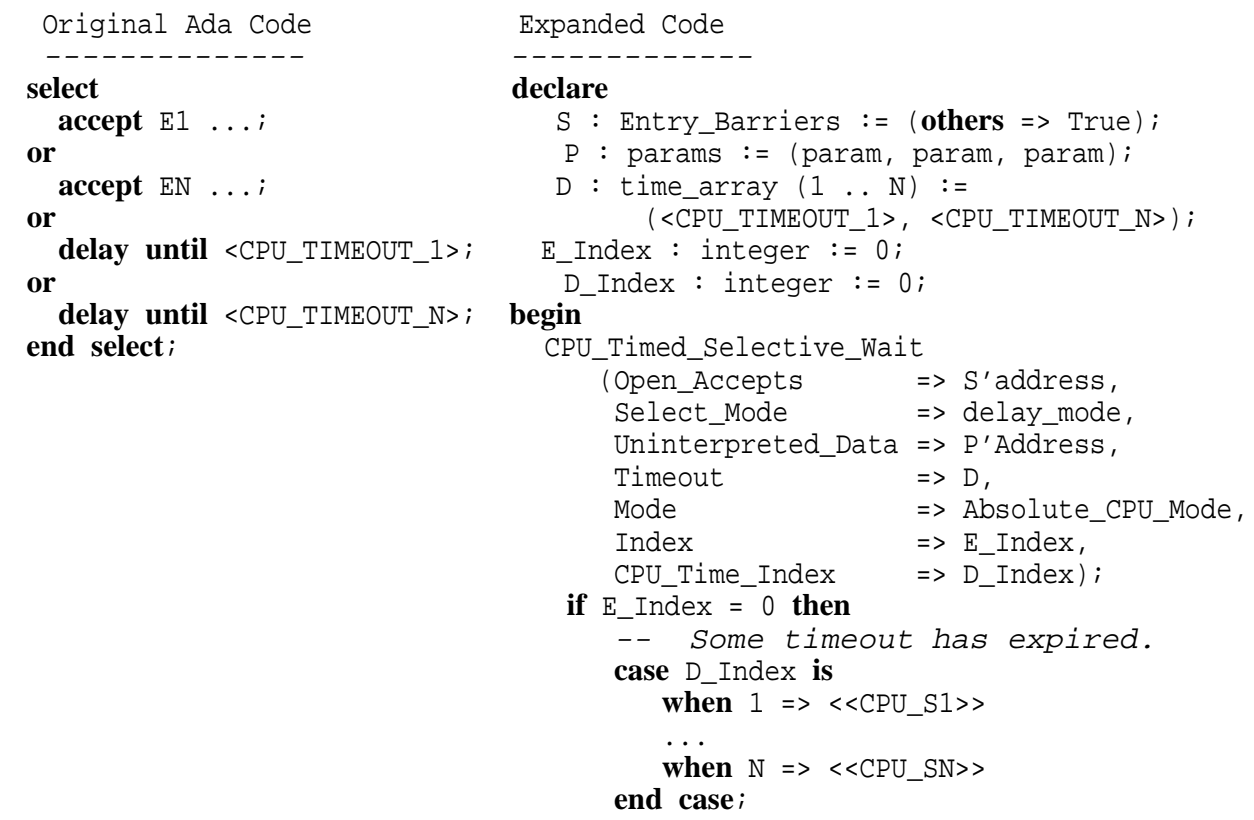

\footnotetext{
3 "If a selective_accept contains more than one delay_alternative, then all shall be delay_relative_statements, or all shall be delay_until_statements for the same time type." ARM95[9], Section 9.7.1(13).
} 


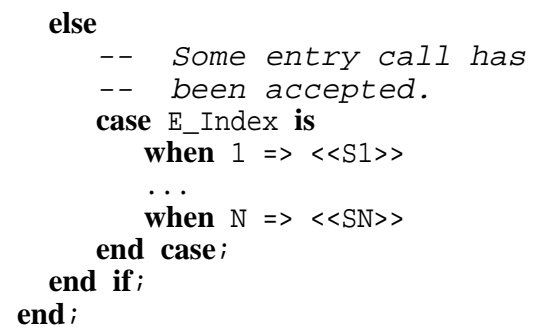

\section{Run-Time}

- Package System.Tasking.Rendezvous. The new CPU_Timed_Selective_Wait subprogram is based on the GNAT Timed_Selective_Wait subprogram. The main differences with the original GNAT version are:

- The data type of the Timeout parameter is an array where all the timeouts specified in the Ada select statement are passed by the front-end.

- Its body calls a variant of the CPU_Timed_Sleep which receives the timeouts array and returns the index of the expired CPU timeout. This index is returned in the CPU_Time_Index parameter. The possible values of the out mode parameters Index and CPU_Time_Index are:

$\begin{array}{lcc} & \text { Index } & \text { CPU_Time_Index } \\ \text { Some entry call was accepted: } & ---- & --------- \\ \text { Some deadline expired: } & 0 & 0 \\ & & \text { <timeout index }>\end{array}$

- Package System.Task_Primitives.Operations. The new variant of the subprogram Ada.CPU_Timed_Sleep calls Ada.CPU_Time.Delays.Multiple_Delay_Until.

- Package Ada.CPU_Time.Delays.Multiple_Delay_Until. This subprogram does the same actions as Delay_Until (described in Section 4.1). However, instead of using a single execution-time timer, it programs as many execution-time timers as the number of delay until alternatives specified by the programmer in the Ada 95 timed selective accept.

In order to identify the execution-time timer which expired, an array of Delay Block registers containing the address of the caller's ATCB and the Timed_Out field is used. When one execution-time timeout expires the unblocked task traverses this array to look for the execution-time timeout which has its Timed_Out field set to True. If no execution-time timer has its Timed_Out field set to true it means that some entry call was accepted, and therefore the task was unblocked by the caller. 


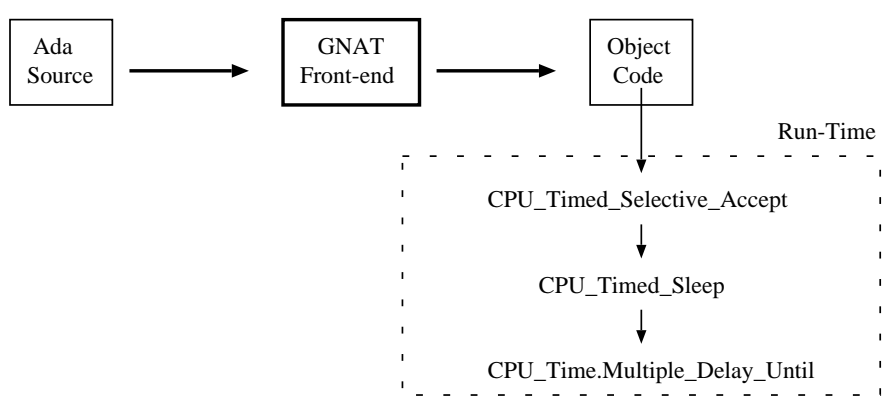

Fig. 2. Run-Time Calls to Implement the CPU Timed Selective Accept.

\section{Discussion}

A prototype implementation has been developed for the execution-time budgeting proposal presented in this paper, using the MaRTE operating system $[1,2]$ that provides a POSIX.13 [8] minimal real-time system interface and includes execution-time clocks and timers. As we have shown, the implementation requires small modifications to the compiler and to the run-time. The modifications were implemented in a short period of time and should not represent a large effort to current compiler implementors. We have compared this implementation with the one presented in [3] defining support for the execution-time clocks and timers as a library package. This latter implementation is very simple, because it does not require modifications to the compiler nor to the runtime system. The overheads are quite similar in both implementations, and are small.

For both approaches, implementations on bare machines or systems without the POSIX execution-time clocks and timers would be a bit more complex because the underlaying execution-time monitoring functionality would have to be implemented in the scheduler. Reference [6] describes one such implementation and it can be seen that it is relatively simple, and that it does not introduce any significant overhead into the scheduler.

Both approaches were discussed at the International Real-Time Ada Workshop in 2002. The conclusion from the Workshop was to recommend the approach presented in this paper, with execution-time functionality integrated in the Ada language, because it provides a simpler model to programmers. However, the group felt that there is a strong need to have the execution-time functionality in Ada, so if the second proposal, with a library implementation, has more probability of success, the group would also recommend its adoption.

Both proposals have been submitted to the Ada Rapporteur Group (ARG) for the inclusion of the execution-time clocks in the next revision of the Ada language. At the time of writing this paper the ARG has expressed preference for the package solution because it has less implementation impact, but a final decision has not been made, and discussions will continue. 


\section{Conclusions}

We have presented a proposal to integrate the POSIX.1 execution-time clocks and timers into the Ada 95 language. This proposal defines a new package named Ada.CPU_Time and describes the modifications done to the GNAT front-end and run-time to allow the use of the execution-time timers in the timed Ada statements. We have also discussed some usage schemes of the Ada.CPU_Time interface.

As a proof of concepts we have modified the GNAT sources to support the executiontime timers with all the Ada 95 timed statements: delay until, timed entry call (to tasks and to protected objects), timed asynchronous transfer of control (ATC). and timed selective accept. We have a modified version of the GNAT compiler which implements all the proposals presented in this paper, and which can be used on top of the MaRTE Operating System [1,2]. Among the different options for implementing execution-time clocks in the Ada language, this proposal represents an easy to use alternative with limited implementation impact. It is now the task of the ARG and the Ada community to decide which of the alternatives is best for inclusion in the next revision of the Ada language.

\section{References}

1. Aldea Rivas M. and González Harbour M. MaRTE OS: Minimal Real-Time Operating System for Embedded Applications Departamento de Electrónica y Computadores. Universidad de Cantabria. http://marte.unican.es/

2. Aldea Rivas M. and González Harbour M. MaRTE OS: An Ada Kernel for Real-Time Embedded Applications. Proceedings of the International Conference on Reliable Software Technologies, Ada-Europe-2001, Leuven, Belgium, Lecture Notes in Computer Science, LNCS 2043, May, 2001, ISBN:3-540-42123-8, pp. 305,316.

3. Aldea Rivas M. and González Harbour M. Extending Ada's Real-Time Systems Annex with the POSIX Scheduling Services. IRTAW-2000, Las Navas, Avila, Spain.

4. Burns A. and Wellings A. Real-Time Systems and Programming Languages. 3rd edition. Addison-Wesley, 2001.

5. Comar, C., Gasperoni, F., and Schonberg, E. The GNAT Project: A GNU-Ada9X Compiler. Technical report. New York University. 1994.

6. González-Harbour M., Aldea Rivas M., Gutiérrez García J.J., Palencia Gutiérrez J.C. Implementing and using Execution-Time Clocks in Ada Hard Real-Time Applications. International Conference on Reliable Software Technologies, Ada-Europa'98, Uppsala, Sweden, in Lecture Notes in Computer Science No. 1411, June, 1998, ISBN:3-540-64563-5, pp. 91, 101.

7. IEEE Std. 1003.1:2001, Information Technology —Portable Operating System Interface (POSIX).

8. IEEE Std. P1003.13-1998, Information Technology — Standarized Application Environment Profile- POSIX Realtime Application Support (AEP). The Institute of Electrical and Electronics Engineers.

9. Intermetrics, Inc. Ada 95 Language Reference Manual. Intermetrics, Inc., Cambridge, Mass., January, 1995.

10. J. Liu, K.J. Lin, W.K. Shih, A. Chang-Shi Yu, J.Y. Chung, and W. Zhao. Algorithms for Scheduling Imprecise Computations. IEEE Computer, pp. 56-68, May 1991. 